\title{
Small RNA biology: From fundamental studies to applications
}

\author{
LI ZhengHe \& ZHOU XuePing* \\ State Key Laboratory of Rice Biology, Institute of Biotechnology, Zhejiang University, Hangzhou 310058, China
}

Received July 10, 2013; accepted July 22, 2013; published online August 9, 2013

Citation: $\quad$ Li Z H, Zhou X P. Small RNA biology: From fundamental studies to applications. Sci China Life Sci, 2013, 56: 1059-1062, doi: 10.1007/s11427-0134535-8

The discovery of RNA silencing as a sequence-specific gene regulation mechanism in eukaryotes has been heralded as the most exciting breakthrough in current biology. The seminal work of Baulcombe and Hamilton in 1999 provided a key insight into the potential function of a tiny RNA species of approximately 25 nucleotides (nt) in RNA silencing in plants [1]. Subsequently, Tuschl and colleagues [2,3] demonstrated that small RNAs of similar size mediated RNA interference (RNAi) in insect and mammalian cells. These discoveries uncovered a previously unrecognized small RNA world and led to a surge in interest in small RNA biology. Now small RNAs are well recognized as ubiquitous, versatile regulators of gene expression in plants, fungi and animals $[4,5]$.

On the basis of their origins, biogenesis and mode of action, small RNAs can be categorized into three major classes: small interfering RNAs (siRNAs), microRNAs (miRNAs) and Piwi-associated interfering RNAs (piRNAs). siRNA is about $21-24 \mathrm{nt}$ in size and is processed from a perfect base-paired double stranded RNA (dsRNA) precursor by RNase III-like enzymes termed Dicers [6]. miRNA is also a cleavage product of Dicer enzymes from an imperfect RNA hairpin formed through inter-molecular base-pairing and is mostly $21 \mathrm{nt}$ long [7]. piRNA is generated in a Dicerindependent manner with longer size (from 24 to $32 \mathrm{nt}$ ) than miRNAs and siRNAs, and is highly enriched in animal germ line cells [8]. Genetic and biochemical studies in Arabidopsis thaliana, Caenorhabditis elegans, Drosophila melanogaster and other model organisms have provided frame-

*Corresponding author (email: zzhou@zju.edu.cn) works of small RNA-mediated regulation mechanisms. In brief, these small RNAs associate with a diverse of proteins from the Argonaute family and form the core RNA silencing processor called RNA-induced silencing complexes (RISCs). Small RNAs guide the RISCs to search for target RNAs by base pairing and cause degradation or translation inhibition of cognate mRNAs, or changes in chromatin structure [9].

Plants are particularly rich in diversity of small RNAs. Thus far several different types of endogenous small RNAs have been identified in plants: trans-acting siRNAs (tasiRNAs), natural antisense transcripts-derived siRNAs (natsiRNAs), heterochromatic siRNAs (hc-siRNAs), long siRNAs (lsiRNAs) and miRNA [10]. These small RNAs participate in virtually every aspect of plant life, such as development timing [11,12], chromatin remodeling [13,14], genome protection [15] as well as responses to biotic and abiotic stresses [16-18].

In plants, small RNAs can also be produced from invading exogenous nucleic acids such as viruses, and these virus-derived siRNAs target cognate RNAs for specific degradation [19,20]. If a recombinant virus harboring a sequence fragment of a plant gene, the cognate endogenous mRNAs can be degraded upon virus infection [21]. This principle inspires scientists to develop a novel reverse genetics tool known as virus-induced gene silencing (VIGS). VIGS represents a simple, fast and transformation-free approach for gene loss-of-function assay, and has been widely used for plant functional genomics, especially in species that are less amenable to traditional genetic manipulation [22-24]. On the other hand, the small RNA-mediated natu- 
ral antiviral response in plants can be reinforced by expressing a sequence of viral origin that potentiates the host RNA silencing defense mechanism [25]. This strategy, which is known as pathogen-derived resistance, has been very successfully utilized in molecular breeding program for viral disease resistance even long before our mechanistic understanding of RNA silencing [26,27]. One outstanding example is the control of Papaya ringspot virus in Hawaii by transgenic expression of viral coat protein gene in papaya plants [28]. While Fire and Mello [29] made the breakthrough discovery in 1998 that dsRNA is the trigger of RNA silencing, Waterhouse et al. [30] showed that transgenic expressing a dsRNA structure derived from a viral sequence effectively induced RNA silencing and conferred specific virus immunity. Since then, this approach has been well adopted for genetic engineering for crop resistance to infections of all major classes of plant viruses [31,32]. In an analogous process termed host-induced gene silencing (HIGS), transgenic expression of dsRNAs in plant triggers robust silencing of cognate mRNAs expressed by invading fungi [33,34], nematode [35,36], parasitic plant [36,37] and even chewing insects [38,39]. HIGS has emerged as a promising strategy to create transgenic germplasm for pest control [40]. In addition, small RNA-based technologies can be harnessed to modify crop agronomic trait to enhance stress tolerance and to increase yields [41,42]. Studies of viral counter-defense measure led to the discovery of viral suppressors of RNA silencing. These suppressors not only facilitate our better understanding of RNA silencing pathway [43-45], but also serve to increase foreign protein production in transgenic plants [46].

In animals, the biogenesis and function of miRNAs have been studied extensively. Animal miRNAs have more relaxed base-pairing requirements and thus regulate greater numbers of target mRNAs than their plant counterparts do. Another different aspect of animal miRNAs is that their targets are often subjected to translation repression instead of RISC-mediated destruction [47]. It is estimated that human genome gives rise to well over 800 mature miRNAs [48]. Each miRNA may regulate hundreds of genes and a majority of genes may be controlled by one or more miRNAs [7]. The normal miRNA activities are needed in proper regulation of many cellular processes ranging from cell differentiation to apoptosis, whereas dysregulation of miRNA expression is associated with various disease conditions [49]. Therefore these miRNAs may serve as novel diagnostic biomarkers [50] and therapeutic targets [51]. One such example is the development of a locked nucleic acid-based antisense oligonucleotide "Miravirsen" that specifically inhibits miR-122 [52], a liver specific miRNA that is required for Hepatitis C virus replication [53]. Report from the Phase IIa clinical trial showed that Miravirsen provided robust dose-dependent anti-viral activity that was maintained beyond the end of therapy [54], highlighting the therapeutic potential of miRNA-based medicine.
Although the diversity and function of endogenous siRNAs in human cells have not been fully delineated, it has been well established that the RNAi machinery can utilize exogenous siRNAs to shut off human gene expression [2]. This has led to the siRNA-based RNAi approach that now has been routinely used in both basic and applied studies [55]. Numerous genome-wide RNAi screenings with synthetic siRNA molecules have begun to systemically identify important genes implicated in various cellular pathways [56]. An important practical consideration is that knocking down the abnormal expression of disease-causing genes by administrated siRNA drugs may offer clinical benefits. By 2011, nearly 30 small RNA-based therapeutic agents had undergone clinical trials for treatment of various diseases, including cancers, physiological and metabolic disorders and viral infections [57,58]. The bench-to-bedside translation of small RNA biology can be anticipated in the near future.

Since the discovery of RNA silencing, research on small RNAs is probably one of the most rapidly advancing fields in the contemporary biology. Numerous efforts have been made to uncover the repertoire and diversity of small RNAs in the past 15 years. This process is greatly expedited by the technical advances in high-throughput deep sequencing of small RNA, leading to an information explosion in the small RNAome [59]. The availability of newly annotated genome sequences also contribute to small RNA profiling through comparative genomics analyses [60,61]. With the everincreasing small RNA species, a major challenging problem is to identify the targets of small RNAs and to annotate their functions. Genetic and biochemical dissection of RNA silencing pathway in model organisms will continue to expand our appreciation for the small RNA-mediated regulatory mechanisms. Improvement of current computational algorithms will likely facilitate our understanding of more comprehensive miRNA targetome [62-64]. New advances in RNA-Seq technologies may enable the experimental examination of changes in target gene expression when miRNA level is deliberately altered [63,65]. Development of methods for real-time monitoring small RNA activities in vivo may provide new insights into their functions in normal physiology and disease [66-68]. Breakthroughs in small RNA delivery methodology could increase its efficacy and minimize side effect [69]. We can anticipate that advances in fundamental small RNA biology will make a big difference in agricultural biotechnology and biomedicine.

This work was supported by the National Natural Science Foundation of China (31100114, 31222004).

1 Hamilton A J, Baulcombe D C. A species of small antisense RNA in posttranscriptional gene silencing in plants. Science, 1999, 286: 950-952

2 Elbashir S M, Harborth J, Lendeckel W, et al. Duplexes of 21-nucleotide RNAs mediate RNA interference in cultured mamma- 
lian cells. Nature, 2001, 411: 494-498

3 Elbashir S M, Lendeckel W, Tuschl T. RNA interference is mediated by 21- and 22-nucleotide RNAs. Gene Dev, 2001, 15: 188-200

4 Aalto A P, Pasquinelli A E. Small non-coding RNAs mount a silent revolution in gene expression. Curr Opin Cell Biol, 2012, 24: 333340

5 Chen X M. Small RNAs - secrets and surprises of the genome. Plant J, 2010, 61: 941-958

6 Zhang C. Novel functions for small RNA molecules. Curr Opin Mol Ther, 2009, 11: 641-651

7 Pasquinelli A E. microRNAs and their targets: recognition, regulation and an emerging reciprocal relationship. Nat Rev Genet, 2012, 13: 271-282

8 Saxe J P, Lin H. Small noncoding RNAs in the germline. Cold Spring Harb Perspect Biol, 2011, 3: a002717

9 Liu Q, Paroo Z. Biochemical principles of small RNA pathways. Annu Rev Biochem, 2010, 79: 295-319

10 Chapman E J, Carrington J C. Specialization and evolution of endogenous small RNA pathways. Nat Rev Genet, 2007, 8: 884-896

11 Chen X M. Small RNAs in development-insights from plants. Curr Opin Genet Dev, 2012, 22: 361-367

12 Van Ex F, Jacob Y, Martienssen R A. Multiple roles for small RNAs during plant reproduction. Curr Opin Plant Biol, 2011, 14: 588-593

13 Olovnikov I, Aravin A A, Fejes Toth K. Small RNA in the nucleus: the RNA-chromatin ping-pong. Curr Opin Genet Dev, 2012, 22: 164-171

14 Simon S A, Meyers B C. Small RNA-mediated epigenetic modifications in plants. Curr Opin Plant Biol, 2011, 14: 148-155

15 Blumenstiel J P. Evolutionary dynamics of transposable elements in a small RNA world. Trends Genet, 2011, 27: 23-31

16 Katiyar-Agarwal S, Jin H L. Role of small RNAs in host-microbe interactions. Annu Rev Phytopathol, 2010, 48: 225-246

17 Lu X Y, Huang X L. Plant miRNAs and abiotic stress responses. Biochem Biophys Res Commun, 2008, 368: 458-462

18 Sunkar R, Chinnusamy V, Zhu J K, et al. Small RNAs as big players in plant abiotic stress responses and nutrient deprivation. Trends Plant Sci, 2007, 12: 301-309

19 Zhu H, Guo H S. The role of virus-derived small interfering RNAs in RNA silencing in plants. Sci China Life Sci, 2012, 55: 119-125

20 Ding S W, Lu R. Virus-derived siRNAs and piRNAs in immunity and pathogenesis. Curr Opinion in Virol, 2011, 1: 533-544

21 Van Kammen A. Virus-induced gene silencing in infected and transgenic plants. Trends Plant Sci, 1997, 2: 409-411

22 Senthil-Kumar M, Mysore K S. New dimensions for VIGS in plant functional genomics. Trends Plant Sci, 2011, 16: 656-665

23 Huang C J, Xie Y, Zhou X P. Efficient virus-induced gene silencing in plants using a modified geminivirus DNA 1 component. Plant Biotechnol J, 2009, 7: 254-265

24 Huang C J, Qian Y J, Li Z H, et al. Virus-induced gene silencing and its application in plant functional genomics. Sci China Life Sci, 2012, 55: 99-108

25 Wilson T M. Strategies to protect crop plants against viruses: pathogen-derived resistance blossoms. Proc Natl Acad Sci USA, 1993, 90 : 3134-3141

26 Lomonossoff G P. Pathogen-derived resistance to plant viruses. Annu Rev Phytopathol, 1995, 33: 323-343

27 Prins M, Laimer M, Noris E, et al. Strategies for antiviral resistance in transgenic plants. Mol Plant Pathol, 2008, 9: 73-83

28 Gonsalves D. Control of papaya ringspot virus in papaya: a case study. Annu Rev Phytopathol, 1998, 36: 415-437

29 Fire A, Xu S, Montgomery M K, et al. Potent and specific genetic interference by double-stranded RNA in Caenorhabditis elegans. Nature, 1998, 391: 806-811

30 Waterhouse P M, Graham M W, Wang M B. Virus resistance and gene silencing in plants can be induced by simultaneous expression of sense and antisense RNA. Proc Natl Acad Sci USA, 1998, 95: 13959-13964

31 Duan C G, Wang C H, Guo H S. Application of RNA silencing to plant disease resistance. Science, 2012, 3: 5

32 Gottula J, Fuchs M. Chapter 5-toward a quarter century of patho- gen-derived resistance and practical approaches to plant virus disease control. Adv Virus Res, 2009, 75: 161-183

33 Pliego C, Nowara D, Bonciani G, et al. Host-induced gene silencing in barley powdery mildew reveals a class of ribonuclease-like effectors. Mol Plant Microbe Interact, 2013, 26: 633-642

34 Nowara D, Gay A, Lacomme C, et al. HIGS: host-induced gene silencing in the obligate biotrophic fungal pathogen blumeria graminis. Plant Cell, 2010, 22: 3130-3141

35 Huang G, Allen R, Davis E L, et al. Engineering broad root-knot resistance in transgenic plants by RNAi silencing of a conserved and essential root-knot nematode parasitism gene. Proc Natl Acad Sci USA, 2006, 103: 14302-14306

36 Fairbairn D J, Cavallaro A S, Bernard M, et al. Host-delivered RNAi: an effective strategy to silence genes in plant parasitic nematodes. Planta, 2007, 226: 1525-1533

37 Tomilov A A, Tomilova N B, Wroblewski T, et al. Trans-specific gene silencing between host and parasitic plants. Plant J, 2008, 56: 389-397

38 Mao Y B, Cai W J, Wang J W, et al. Silencing a cotton bollworm P450 monooxygenase gene by plant-mediated RNAi impairs larval tolerance of gossypol. Nat Biotechnol, 2007, 25: 1307-1313

39 Mao Y B, Tao X Y, Xue X Y, et al. Cotton plants expressing CYP6AE14 double-stranded RNA show enhanced resistance to bollworms. Transgenic Res, 2011, 20: 665-673

40 Runo S. Engineering host-derived resistance against plant parasites through RNA interference: challenges and opportunities. Bioeng Bugs, 2011, 2: 208-213

41 Auer C, Frederick R. Crop improvement using small RNAs: applications and predictive ecological risk assessments. Trends Biotechnol, 2009, 27: 644-651

42 Macovei A, Gill S S, Tuteja N. microRNAs as promising tools for improving stress tolerance in rice. Plant Signal Behav, 2012, 7: 1296-1301

43 Jiang L, Wei C H, Li Y. Viral suppression of RNA silencing. Sci China Life Sci, 2012, 55: 109-118

44 Wu Q, Wang X, Ding S W. Viral suppressors of RNA-based viral immunity: host targets. Cell Host Microbe, 2010, 8: 12-15

45 Wu J G, Wang C Z, Du Z G, et al. Identification of Pns12 as the second silencing suppressor of Rice gall dwarf virus. Sci China Life Sci, 2011, 54: 201-208

46 Saxena P, Hsieh Y C, Alvarado V Y, et al. Improved foreign gene expression in plants using a virus-encoded suppressor of RNA silencing modified to be developmentally harmless. Plant Biotechnol J, 2011, 9: 703-712

47 Djuranovic S, Nahvi A, Green R. A parsimonious model for gene regulation by miRNA. Science, 2011, 331: 550-553

48 Berezikov E, Guryev V, van de Belt J, et al. Phylogenetic shadowing and computational identification of human microRNA genes. Cell, 2005, 120: 21-24

49 Zhang C. Micrornomics: a newly emerging approach for disease biology. Physiol Genomics, 2008, 33: 139-147

50 Weiland M, Gao X H, Zhou L, et al. Small RNAs have a large impact: circulating microRNAs as biomarkers for human diseases. RNA Biol, 2012, 9: 850-859

51 Jackson A L, Levin A A. Developing microRNA therapeutics: approaching the unique complexities. Nucleic Acid Ther, 2012, 22: 213-225

52 Elmen J, Lindow M, Schutz S, et al. LNA-mediated microRNA silencing in non-human primates. Nature, 2008, 452: 896-899

53 Li Y, Masaki T, Lemon S M. miR-122 and the hepatitis C RNA genome: more than just stability. RNA Biol, 2013, 10: 907-916

54 Janssen H L, Reesink H W, Lawitz E J, et al. Treatment of HCV infection by targeting microRNA. N Engl J Med, 2013, 368: 16851694

55 Snead N M, Rossi J J. Biogenesis and function of endogenous and exogenous siRNAs. Wiley Interdiscip Rev RNA, 2010, 1: 117-131

56 Mohr S, Bakal C, Perrimon N. Genomic screening with RNAi: results and challenges. Annu Rev Biochem, 2010, 79: 37-64

57 Burnett J C, Rossi J J, Tiemann K. Current progress of siRNA/ 
shRNA therapeutics in clinical trials. Biotechnol J, 2011, 6: 11301146

58 Davidson B L, McCray P B Jr. Current prospects for RNA interference-based therapies. Nat Rev Genet, 2011, 12: 329-340

59 Ozsolak F, Milos P M. RNA sequencing: advances, challenges and opportunities. Nat Rev Genet, 2011, 12: 87-98

60 Lister R, Gregory B D, Ecker J R. Next is now: new technologies for sequencing of genomes, transcriptomes, and beyond. Curr Opin Plant Biol, 2009, 12: 107-118

61 Zuo J, Wang Y, Liu H, et al. microRNAs in tomato plants. Sci China Life Sci, 2011, 54: 599-605

62 Thomas M, Lieberman J, Lal A. Desperately seeking microRNA targets. Nat Struct Mol Biol, 2010, 17: 1169-1174

63 Yu J, Wang F. Recent progress in microRNA study: benefits from technique advance. Sci China Life Sci, 2012, 55: 649-650

64 John B, Sander C, Marks D S. Prediction of human microRNA tar- gets. Methods Mol Biol, 2006, 342: 101-113

65 Muniategui A, Pey J, Planes F J, et al. Joint analysis of miRNA and mRNA expression data. Brief Bioinform, 2013, 14: 263-278

66 Mullokandov G, Baccarini A, Ruzo A, et al. High-throughput assessment of microRNA activity and function using microRNA sensor and decoy libraries. Nat Methods, 2012, 9: 840-846

67 Mansfield J H, Harfe B D, Nissen R, et al. MicroRNA-responsive 'sensor' transgenes uncover hox-like and other developmentally regulated patterns of vertebrate microRNA expression. Nat Genet, 2004, 36: 1079-1083

68 Fellmann C, Zuber J, McJunkin K, et al. Functional identification of optimized RNAi triggers using a massively parallel sensor assay. Mol Cell, 2011, 41: 733-746

69 Whitehead K A, Langer R, Anderson D G. Knocking down barriers: advances in siRNA delivery. Nat Rev Drug Discov, 2009, 8: 129138

Open Access This article is distributed under the terms of the Creative Commons Attribution License which permits any use, distribution, and reproduction in any medium, provided the original author(s) and source are credited. 